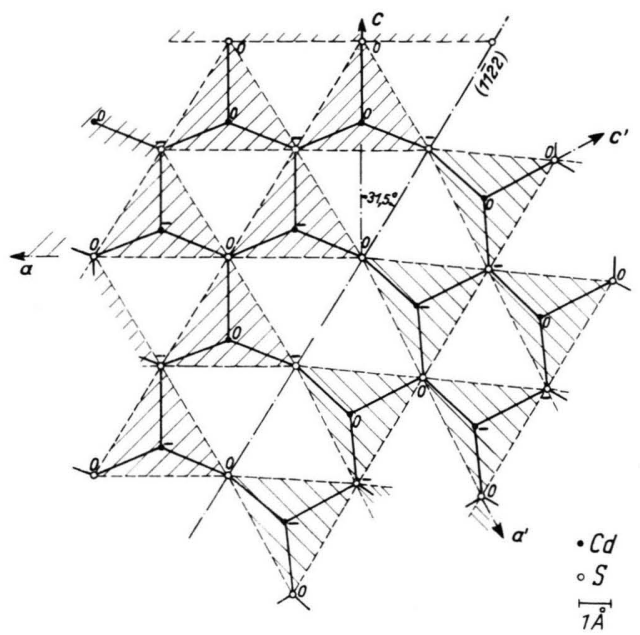

Abb. 3a. $31^{0}$-Zwilling. Atome mit - liegen um $0,97 \AA$ hinter der Zeichenebene. Die von der Zwillingsebene geschnittenen Tetraeder fallen aus.

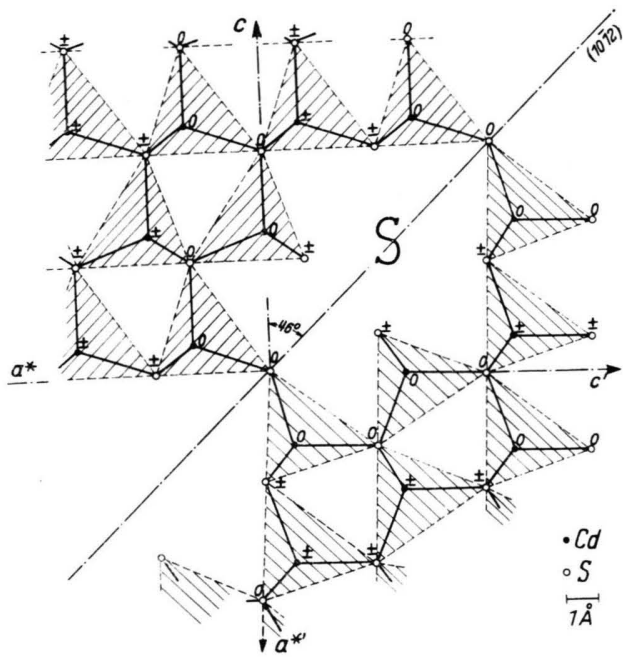

Abb. 3b. $46^{0}$-Zwilling.

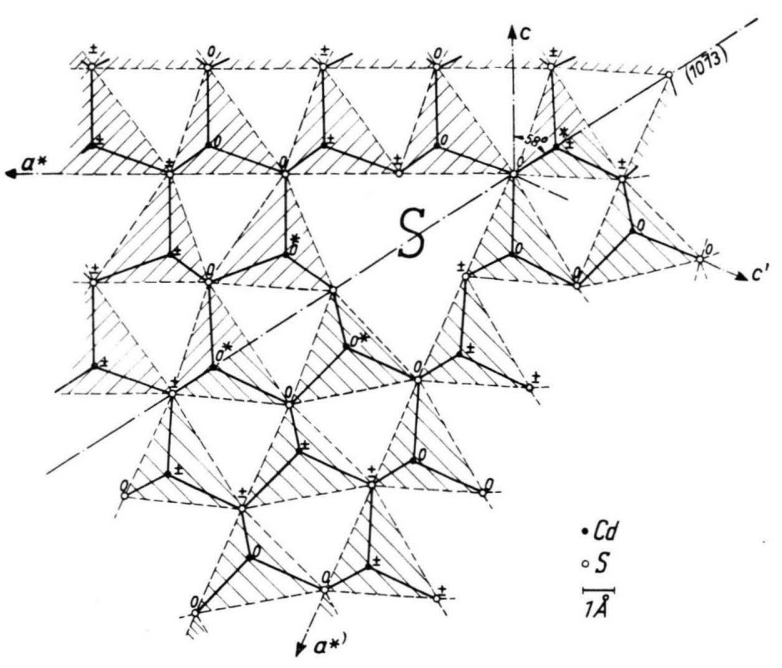

Abb. 3c. 58 -Zwilling. Atome mit \pm sind in der Projektion zusammenfallende Atome, die 2,07 $\AA$ vor und hinter der Zeichenebene liegen. Die durch * gekennzeichneten Tetraeder sind leicht deformiert. Die an der Zwillingsebene bestehenden Lücken (S) sind durch Fremdatome und unregelmäßig gelagerte $[\mathrm{CdS}]_{4}$-Tetraeder ausgefüllt $\mathrm{zu}$ denken.

Abb. 3 a-c. Darstellung der Tetraederverknüpfung in Schnitten senkrecht zur Zwillingsebene. Die Spur der Zwillingsebene und die Achsen der beiden Hälften sind eingezeichnet. Mit 0 bezeichnete Atome liegen in der Zeichenebene.

Die Verzerrung der Tetraeder dürfte dabei infolge der hohen Bildungstemperatur energetisch keine Schwierigkeiten bedeuten.

Für Diskussionen danke ich Frau Dr. Dornberger und Herrn Prof. Kleber.

\title{
Zur Definition der Bindungsordnung in der Quantenchemie
}

\author{
Von W. BINGEL
}

Aus der Forschungsstelle für Spektroskopie in der Max-Planck-Gesellschaft, Hechingen

(Z. Naturforschg. 9a, 436-439 [1954]; eingegangen am 25. Februar 1954)

Die beiden Definitionen der Bindungsordnung nach Pauling, Brockway und Beach und nach Penney werden miteinander verglichen. Es wird gezeigt, da $\beta$ die erstere bei geeigneter Berücksichtigung des Überlappungsintegrals zwischen zwei Valenzstrukturen mit der letzteren identisch wird.

$\mathrm{D}$ ie Ordnung einer Bindung zwischen zwei Atomen eines Moleküls ist ein wichtiger Begriff in der Quantenchemie. Er wurde geschaffen, um die Eigenschaften der Bindungen in ungesättigten und aromatischen Molekülen, die ja keine reinen Ein- fach- oder Doppelbindungen sind, zu kennzeichnen. Indem man einer isolierten $\mathrm{C}=\mathrm{C}$-Bindung vom Atomabstand 1,33 $\AA$ die Bindungsordnung 2, einer isolierten C-C-Bindung vom Atomabstand 1,54 $\AA$ die Ordnung 1 zuschreibt, erwartet man für die 
L. Herforth und J. Kr u mbiegel, Beobachtungen an CdS-Kristallen nach Untersuchungen im Mikroskop und Leitfähigkeitsmessungen (S. 432).
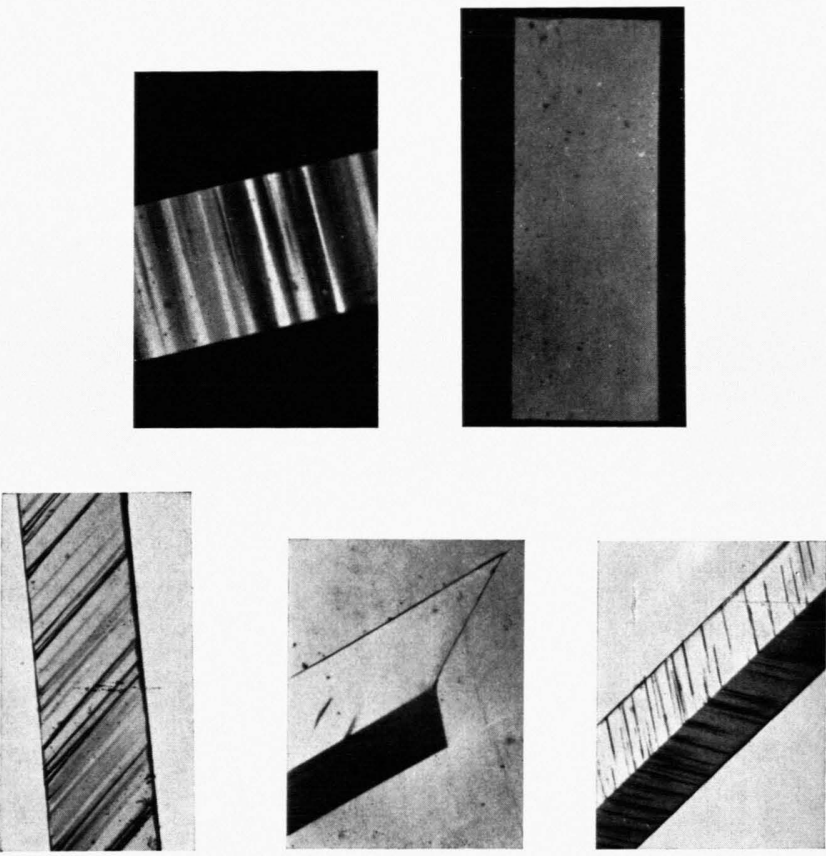

Abb. 2. Einige CdS-Kristalle im polarisierten Licht.

K.-H. J o st, Über die Lage der Zwillingsebenen in hexagonalen CdS-Kristallen (S. 435).

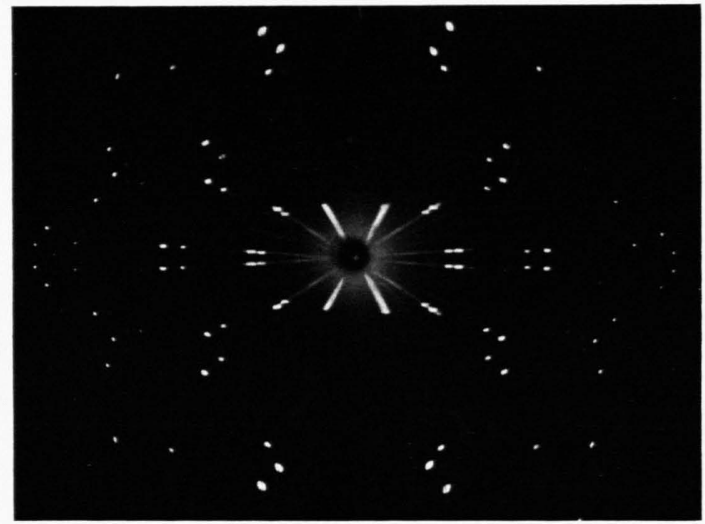

Abb. 2. Schwenkaufnahme eines CdS-Zwillingskristalls mit Cu-Strahlung, Drehachse senkrecht zur Zwillingsebene. 

Ordnung $P$ einer $(\mathrm{C}-\mathrm{C})_{\text {arom. }}$-Bindung in konjugierten Molekülen, deren Atomabstand $l$ zwischen den Werten für die Einfach- und Doppelbindung liegt, ein Wert zwischen 1 und 2. Hat man $P$ in der Theorie definiert, so kann man aus dem Zusammenhang $l=f(P)$ Atomabstände theoretisch berechnen und mit dem Experiment vergleichen. Die Funktion $f(P)$ kann dabei entweder aus der Theorie entnommen werden ${ }^{1}$ oder durch einige Punkte $l_{\text {exp. }}, P_{\text {theor. }}$ empirisch festgelegt werden ${ }^{2}$. Auf diese Weise ist heute eine theoretische Bestimmung der Atomabstände in kondensierten Kohlenwasserstoffen mit einem mittleren Fehler von $\pm 0,02 \AA$ möglich. Abb. 1 ,

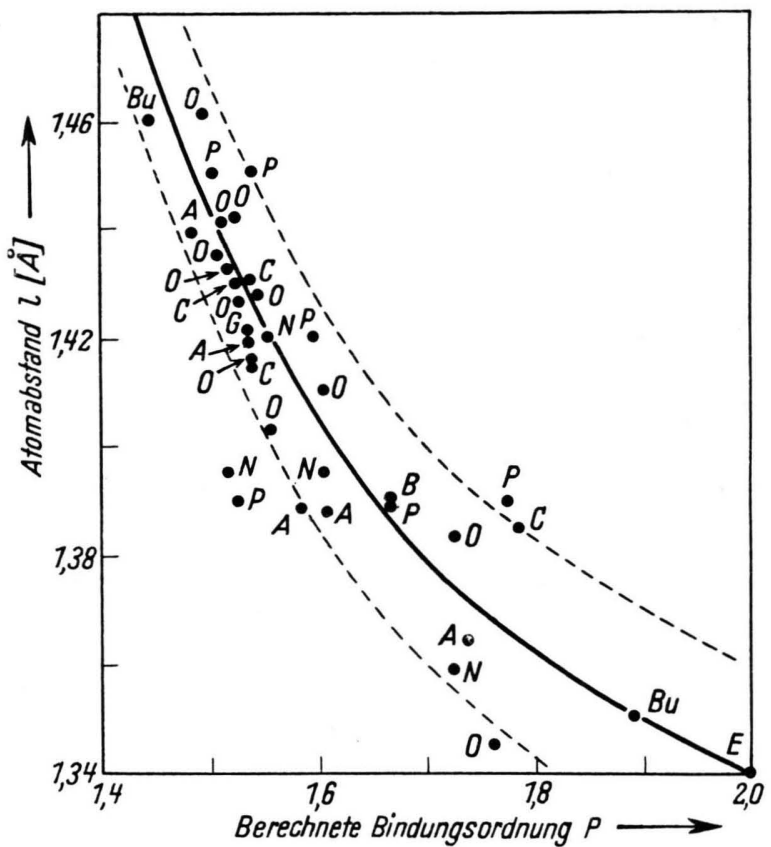

Abb. 1. Berechnete Bindungsordnungen $P$ mit MOMethode als Funktion der beobachteten Atomabstände $l$ in $\AA$ in konjugierten Kohlenwasserstoffen. $\mathrm{A}=$ Anthracen, $\mathrm{Be}=\mathrm{Benzol}, \mathrm{Bu}=\mathrm{Butadien}, \mathrm{C}=$ Coronen, $\mathrm{E}=$ Äthylen, $\mathrm{G}=$ Graphit, $\mathrm{N}=$ Naphthalin, $\mathrm{O}=$ Ovalen, $\mathrm{P}=$ Pyren.

die einer zusammenfassenden Darstellung von Coulson entnommen ist ${ }^{3}$, zeigt eine Zusammenstellung der bis 1951 vorhandenen Resultate. Die ausgezogene Kurve entspricht $l=f(P)$, die beiden gestrichelten Kurven zu beiden Seiten in einem Ab-

1 C. A. Coulson, Proc. Roy. Soc. A 169, 413 [1939].

${ }^{2}$ L. Pauling, L. O. Brockway u. I. Y. Beach, J. Amer. Chem. Soc. 57, 2705 [1953].

3 C. A. Coulson, Proc. Roy. Soc. A 207, 91 [1951]. stand von $0,02 \AA$ geben den oben erwähnten Fehlerbereich.

Die Bindungsordnung $P$ läßt sich gemäß $P=1+p$ in zwei Anteile zerlegen, deren erster den $\sigma$-Elektronen und deren zweiter den $\pi$-Elektronen des Moleküls entspricht (mobile bond order). Die Bindungsordnung $p$ der $\pi$-Elektronen für die Bindung zwischen zwei Atomen $r$ und $s$ ist in der ,Methode der Molekülbahnen" (molecular orbital method) - der einen der beiden wichtigsten Rechenmethoden der Quantenchemie - eindeutig definiert gemäß

$$
p_{r s}=\sum_{i} n_{i} c_{r i} c_{s i}
$$

wobei $c_{r i}$ und $c_{s i}$ die Koeffizienten der „Atombahnen" (atomic orbital $=\mathrm{AO}$ ) des $r$-ten bzw. $s$-ten Atoms in der $i$-ten ,Molekülbahn“ (molecular orbi$t a l=\mathrm{MO})$ sind und $n_{i}$ die Besetzungszahl derselben ist.

In der zweiten Rechenmethode der Quantenchemie, der "Methode der Valenzstrukturen“ (valence bond method) gibt es dagegen im wesentlichen zwei verschiedene Definitionen für $p_{r s}$. Zum Verständnis des folgenden seien die Grundformeln dieser Methode kurz zusammengestellt ${ }^{4}$. Die Wellenfunktion $\psi$ der $\pi$-Elektronen des Moleküls wird als Linearkombination von Valenzfunktionen $\psi_{\alpha}$ angesetzt

$$
\psi=\sum_{\alpha=1}^{\nu} a_{\alpha} \psi_{\alpha} .
$$

Jedes $\psi_{\alpha}$ entspricht dabei einer ganz bestimmten Anordnung der $\pi$-Bindungen im Molekül und damit einer bestimmten Valenzstruktur (s. Abb. 2). Die Energie der $\pi$-Elektronen berechnet sich dann ${ }^{5}$ aus

$$
E=\frac{\int \psi^{*} H \psi \mathrm{d} \tau}{\int \psi^{*} \psi \mathrm{d} \tau}=\frac{\sum_{\alpha} \sum_{\beta} a_{\alpha} a_{\beta} H_{\alpha \beta}}{\sum_{\alpha} \sum_{\beta} a_{\alpha} a_{\beta} S_{\alpha \beta}}
$$

mit $H_{\alpha \beta}=\int \psi_{\alpha}^{*} H \psi_{\beta} \mathrm{d} \tau, S_{\alpha \beta}=\int \psi_{\alpha}^{*} \psi_{\beta} \mathrm{d} \tau$,

indem $E$ bezüglich der $a_{\alpha}$ zu einem Minimum gemacht wird. Aus der Bedingung $\partial E / \partial a_{\alpha}=0 \quad(\alpha=1,2 . . v)$ folgt das System linearer Gleichungen

$$
\sum_{\beta}\left(H_{\alpha \beta}-E S_{\alpha \beta}\right) a_{\beta}=0 \quad(\alpha=1,2 \ldots v)
$$

für die Koeffizienten $a_{\beta}$ und als Lösbarkeitsbedingung von (4) die Säkulargleichung

$$
\operatorname{det}\left|H_{\alpha \beta}-E S_{\alpha \beta}\right|=0
$$

${ }^{4}$ W. Moffit, Proc. Roy. Soc. A 199, 487 [1949].

5 Da die $\psi_{\alpha}$ reelle Funktionen sind, können auch die Koeffizienten $a_{\alpha}$ als reell angenommen werden. 
für die Energie $E$. Jede Wurzel $E^{\tau}$ von (5) ergibt in (4) eingesetzt - ein System von $a_{\alpha}^{\tau}$, die durch (4) bis auf einen gemeinsamen Faktor festgelegt sind. Dieser Faktor schließlich bestimmt sich aus der Normierung von $\psi$

$$
\int \psi^{*} \psi \mathrm{d} \tau=\sum_{\alpha, \beta} a_{\alpha} a_{\beta} S_{\alpha \beta}=1 .
$$

Die Matrixelemente des Hamilton-Operators $H$ (die $H_{\alpha \beta}$ ) und von Eins (die $S_{\alpha \beta}$ ) lassen sich für Singulettstrukturen nach von Pauling angegebenen Regeln ${ }^{6}$ leicht ermitteln; man erhält bei Beschränkung auf einfache Austauschintegrale für $H_{\alpha \beta}$ und Vernachlässigung der Überlappung zwischen benachbarter $\mathrm{AO}^{\prime}$ 's für $S_{\alpha \beta}$

$$
\begin{aligned}
& H_{\alpha \beta}=S_{\alpha \beta}\left(Q+\sum_{r>s} \gamma_{\alpha, \beta}^{r, s} \cdot J_{r s}\right), \\
& S_{\alpha \beta}=2^{i-N} .
\end{aligned}
$$

Dabei ist $Q$ das Coulomb-Integral, $J_{r s}$ das einfache Austauschintegral zwischen den $\mathrm{AO}^{\prime}$ s $r$ und $s ; \gamma_{\alpha, \beta}^{r, s}$ eine Zahl, die sich aus der Überlagerungsstruktur der Strukturen $\alpha$ und $\beta$ ergibt gemä $\beta$

$$
\gamma_{\alpha, \beta}^{r, s}=1(-2) \text {, }
$$

wenn das Atompaar $r, s$ in der gleichen Insel der Überlagerungsstruktur liegt und um eine ungerade (gerade) Zahl von Bindungen getrennt ist;

$$
\gamma_{\alpha, \beta}^{r, s}=-\frac{1}{2},
$$

wenn $r$ und $s$ in verschiedenen Inseln liegen. Die Zahl der Inseln sei $i$, die $\pi$-Elektronenzahl $2 N$. Zur Erläuterung dieser Regeln zeigt Abb. 2 die beiden Kékulé-Strukturen I und II des Benzols und deren Überlagerungsstrukturen.

Pauling, Brockway und Beach ${ }^{2}$ definieren nun die Bindungsordnung durch

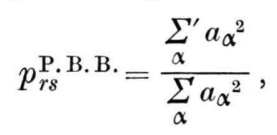

wobei die Zähler-Summe nur über diejenigen Strukturen $\alpha$ läuft, bei denen $r$ und $s$ durch einen Valenzstrich verbunden sind, die Nenner-Summe läuft dagegen über alle $\alpha$ und ist nur bei noch nicht normierten Koeffizienten erforderlich. Nun haben wir aber gesehen, daß die Normierung von $\psi$ - und zwar schon bei Vernachlässigung der Überlappung benachbarter $A O^{\prime} s$ - auf die Normierungsbedingung (6) führt und nicht auf

\footnotetext{
${ }^{6}$ L. Pauling, J. Chem. Phys. 1, 280 [1933].
}

$$
\sum_{\alpha} a_{\alpha}^{2}=1,
$$

was man aus (6) durch Vernachlässigung von $S_{\alpha \beta}$ für $\alpha \neq \beta$ erhält, $S_{\alpha \alpha}=1$ für alle $\alpha$. Man muß daher die Überlappungsintegrale $S_{\alpha \beta}$ zwischen zwei Strukturen - da man sie in der Säkulargleichung (5) mitnimmt - konsequenterweise auch in der Normierungsbedingung und damit auch in der Definition der Bindungsordnung berücksichtigen und wird an Stelle von (8) für $p_{r s}$ einen Ausdruck von der Form

$$
p_{r s}=\frac{\sum_{\alpha, \beta} a_{\alpha} a_{\beta} \cdot S_{\alpha \beta} \cdot \varphi_{\alpha, \beta}^{r, s}}{\sum_{\alpha, \beta} a_{\alpha} a_{\beta} S_{\alpha \beta}}
$$

erwarten, wobei der aus dieser Überlegung allein nicht erschließbare Faktor $\varphi_{\alpha, \beta}^{r, s}$ im Zähler dieses Ausdruckes der Beschränkung der Summation im Zähler von (8) auf Strukturen mit einem Valenzstrich zwischen $r$ und $s$ entspricht.

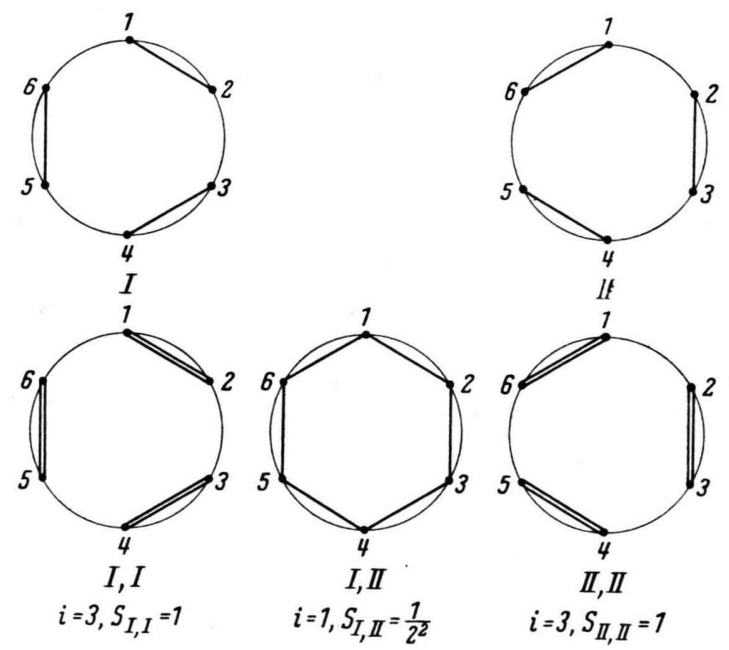

Abb. 2. Die Kékulé-Strukturen I und II des Benzols und ihre Überlagerungsstrukturen. Die Zahl der Inseln $i$ und das nach (7) ermittelte $S_{a \beta}$ sind unter jeder Überlagerungsstruktur angegeben. Die Zahl der $\pi$-Elektronen ist beimBenzol $2 N=6$, je eins von jedem C-Atom.

Im folgenden wird gezeigt, daß die von Penne ${ }^{7}$ eingeführte Bindungsordnung, die im allgemeinen eine bessere Übereinstimmung mit dem Experiment ergibt und daher auch in der Literatur der Pauling schen vorgezogen wird, sich so umformen läßt, daß sie die Gestalt $\left(8^{\prime}\right)$ mit einer sinnvollen Wahl von $\varphi_{\alpha, \beta}^{r, s}$ annimmt. Einsetzen von (7) in (3a) gibt unter Berücksichtigung von (6) den folgenden Ausdruck für die Energie der $2 \mathrm{~N} \pi$-Elektronen ${ }^{4}$

\footnotetext{
${ }^{7}$ W. G. Penney, Proc. Roy. Soc. A 158, 306 [1937].
} 


$$
\begin{aligned}
& E=Q+\sum_{r>s} c_{r s}(a) \cdot J_{r s}, \\
& c_{r s}(a)=\sum_{\alpha} \sum_{\beta} a_{\alpha} \alpha_{\beta} S_{\alpha \beta} \cdot \gamma_{\alpha, \beta}^{r, s} .
\end{aligned}
$$

Vergleicht man dies mit der Lösung des $2 N$-Elektronenproblems durch das Diracsche Vektormodell ${ }^{8,9}$

$$
E=Q-\frac{1}{2} \sum_{r>s}\left(1+4 \overline{\boldsymbol{s}_{r} \cdot \boldsymbol{s}_{s}}\right) \cdot J_{r s}
$$

wobei $\overline{\boldsymbol{s}_{r} \cdot \boldsymbol{s}_{s}}$ den Mittelwert der gegenseitigen Orientierung der Spinvektoren der $\pi$-Elektronen bei den Atomen $r$ und $s$ darstellt, so erhält man

$$
c_{r s}=-\frac{1}{2}\left(1+4 \overline{s_{r} \cdot s_{s}}\right) .
$$

Nun ist die Penneysche Definition der Bindungsordnung?

$$
p_{r s}^{\text {Penney }}=-\frac{4}{3} \cdot \overline{\boldsymbol{s}_{r} \cdot \boldsymbol{s}_{s}}
$$

und aus (9), (11) und (12) ergibt sich

$$
\begin{aligned}
p_{r s}^{\text {Penney }}=\frac{1}{3}\left(1+2 c_{r s}\right) & =\frac{1}{3}\left(1+2 \sum_{\alpha, \beta} a_{\alpha} \alpha_{\beta} S_{\alpha \beta} \cdot \gamma_{\alpha, \beta}^{r, 8}\right) \\
= & \sum_{\alpha, \beta} a_{\alpha} \alpha_{\beta} S_{\alpha \beta} \cdot \varphi_{\alpha, \beta}^{r, s}, \\
\varphi_{\alpha, \beta}^{r, 8}=\frac{1}{3} & \left(1+2 \gamma_{\alpha, \beta}^{r, s}\right),
\end{aligned}
$$

also ein Ausdruck von der Form ( $\left.8^{\prime}\right)$ mit der speziellen, durch (13a) gegebenen Funktion $\varphi_{\alpha, \beta}^{r, s}$. Aus den $\mathrm{P}$ a u ling schen Regeln für $\gamma_{\alpha, \beta}^{r, s}$ erhält man

$\varphi_{\alpha, \beta}^{r, 8}=\left\{\begin{array}{l}0, \text { wenn } r \text { und } s \text { in verschiedenen Inseln der } \\ \text { Überlagerungsstruktur liegen } \\ +1(-1), \text { wenn } r \text { und } s \text { in der gleichen } \\ \text { Insel liegen und eine ungerade (gerade) } \\ \text { Zahl von Bindungen voneinander entfernt } \\ \text { sind. }\end{array}\right.$

Zusammengefaßt werden (13) und (14) in

$$
p_{r s}=\sum_{\alpha, \beta}^{\prime} a_{\alpha} a_{\beta} S_{\alpha \beta}-\sum_{\alpha, \beta}^{\prime \prime} a_{\alpha} \alpha_{\beta} S_{\alpha \beta},
$$

wobei die erste (zweite) Summe über alle Strukturpaare $\alpha, \beta$ zu erstrecken ist, für die die Atome $r$ und $s$ in der gleichen Insel der Überlagerungsstruktur von $\alpha$ und $\beta$ liegen und durch eine ungerade (gerade)

${ }^{8}$ J. H. van Vleck, Phys. Rev. 45, 405 [1934].
Zahl von Bindungen voneinander getrennt sind. Bei aromatischen Kohlenwasserstoffen mit einer geraden Zahl von Ringatomen und bei Beschränkung auf nächste Nachbarn - diese Einschränkung brauchte bisher nicht gemacht zu werden - fällt die zweite Summe völlig weg.

Die nach (15) berechnete Bindungsordnung ist im Gegensatz zu (8) - unabhängig von der Auswahl des Systems der $v$ linear unabhängigen Strukturen, da dies für das Penneysche $p$ gilt. Ferner spricht für (15) noch die Tatsache, daß sich hieraus die Bindungsordnungen unter Umständen einfacher berechnen lassen als aus (12). Wenn man nämlich, wie dies bei kondensierten Kohlenwasserstoffen meistens geschieht, alle Austauschintegrale zwischen nächsten Nachbarn als gleich annimmt und diejenigen zwischen nicht benachbarten Atomen vernachlässigt, so vereinfacht sich (10) zu

$$
E=Q-\frac{k}{2} J+\frac{3}{2} J \cdot \sum_{r>8} p_{\mathrm{rs}}=Q-\frac{k}{2} J+\frac{3}{2} J k \bar{p} .
$$

Dabei ist $J$ das Austauschintegral zwischen nächsten Nachbarn und $k$ die Zahl benachbarter Atompaare im Molekül. Man erhält in diesem Fall aus der Penneyschen Formel nur einen Mittelwert über alle Bindungsordnungen des Moleküls. Um die $p_{r s}$ einzeln zu erhalten, muß man zusätzlich eine Störungsrechnung durchführen ${ }^{7}$, in der das Austauschintegral für dasjenige Atompaar, für das man $p_{r s}$ bestimmen will, einen von allen anderen Austauschintegralen verschiedenen Wert hat und hierfür die Säkulargleichung lösen. Und zwar hat man dies so oftmals durchzuführen, als die Zahl der voneinander verschiedenen $p_{r s}$ - die man schon aus der Symmetrie des betrachteten Moleküls entnehmen kannbeträgt. Mit der neuen Definition (15) dagegen ist diese Störungsrechnung nicht erforderlich, alle $p_{r s}$ lassen sich mit den für lauter gleiche Austauschintegrale ermittelten $a_{\alpha}$ bestimmen.

${ }^{9}$ R. Serber, Phys. Rev. 45, 461 [1934]. 